\title{
Progressive Disease by Computed Tomography
}

National Cancer Institute

\section{Source}

National Cancer Institute. Progressive Disease by Computed Tomography. NCI

Thesaurus. Code C123606.

There is an increase in the size and extent of tissue involvement by cancer, based on CT scan and criteria-defined assessments. 